\title{
Energy Scaling of Yellow Emission from Monolithic Diamond Raman Lasers
}

\author{
Sean Reilly, Vasili G. Savitski, Hangyu Liu, Erdan Gu, Martin D. Dawson, Alan J. Kemp \\ Institute of Photonics, Dept. of Physics, University of Strathclyde, Glasgow, Scotland, G1 1RD
}

Recent advances in the growth of low loss single crystal diamond [1] coupled with its high Raman gain and high thermal conductivity have led to the material becoming an established Raman laser material. With applications often having to adapt to available laser wavelengths, Raman lasers can be used as a simple means to shift ubiquitous commercial laser sources to the hard to reach but application rich spectral regions. The authors recently demonstrated a compact, robust monolithic diamond Raman laser shifting $20 \mu \mathrm{J}$ nanosecond pulses from a Q-switched $532 \mathrm{~nm}$ laser to Raman wavelengths of $573 \mathrm{~nm}, 620 \mathrm{~nm}$ and $676 \mathrm{~nm}$ with a conversion efficiency of $84 \%$. This work investigates the energy scalability of such a system.

A significant increase in the output pulse energy is reported under pulsed pumping at $532 \mathrm{~nm}$. Two devices were tested. Firstly, a $2 \mathrm{~mm}$ long plano-convex diamond device was implemented, with microlens structures etched onto one surface. When mirror coated, the $13 \mathrm{~mm}$ radius of curvature $(\mathrm{RoC})$ structure (similar to the one described in [2]) forms a stable cavity with the opposite diamond face, resulting in a TEM $_{00}$ mode with a radius of $24 \mu \mathrm{m}$. Secondly, a $2 \mathrm{~mm}$ long plane - plane device, again mirror coated on each face, was explored.

The microlensed system was pumped with an Elforlight FQS laser, delivering near diffraction limited pulses of $<5 \mathrm{~ns}$ duration at energies of $155 \mu \mathrm{J}$ at a pump spot radius of $23 \mu \mathrm{m}$. A maximum Raman pulse energy of $92 \mu \mathrm{J}$ was achieved (Fig 1a) - a sevenfold improvement on the 13.6 $\mu \mathrm{J}$ achieved in [2] - consisting of approximately $60 \mu \mathrm{J}$ of $1^{\text {st }}$ Stokes and $32 \mu \mathrm{J}$ of $2^{\text {nd }}$ Stokes emission (Fig.1(a)). With a cavity mode defined predominantly by the RoC of the microlens, further power scaling of this device is restricted by limitations in the fabrication of longer $\mathrm{RoC}$ lenses in order to avoid optical damage to the coatings. With a plane - plane device, however, the cavity mode is less constrained. It is therefore possible to power scale simply by increasing the pump spot radius. Pumping with a Continuum Minilite II laser at a pump spot radius of $195 \mu \mathrm{m}, 3.1 \mathrm{~mJ}$ of Raman output power was achieved at a pump pulse energy of $8 \mathrm{~mJ}$ (Fig.1(b)), a 300 fold increase in Raman output power when compared with the results presented in [2]. The Raman pulse durations were slightly shorter than the pump pulse durations, as expected. First Stokes pulse durations of approximately $2 \mathrm{~ns}$ and $5 \mathrm{~ns}$ were measured at rep rates of $10 \mathrm{kHz}$ and $10 \mathrm{~Hz}$ for the microlens and plane - plane devices, respectively. The $\mathrm{M}^{2}$ of the microlens and plane - plane devices ( $1^{\text {st }}$ Stokes) were measured to be 8 and 10 , respectively. The effect of the pump beam size on the Raman output beam profile will be presented and analysed. The results cannot be fully explained by the previously hypothesized simple model based on Raman gain guiding [3,4].
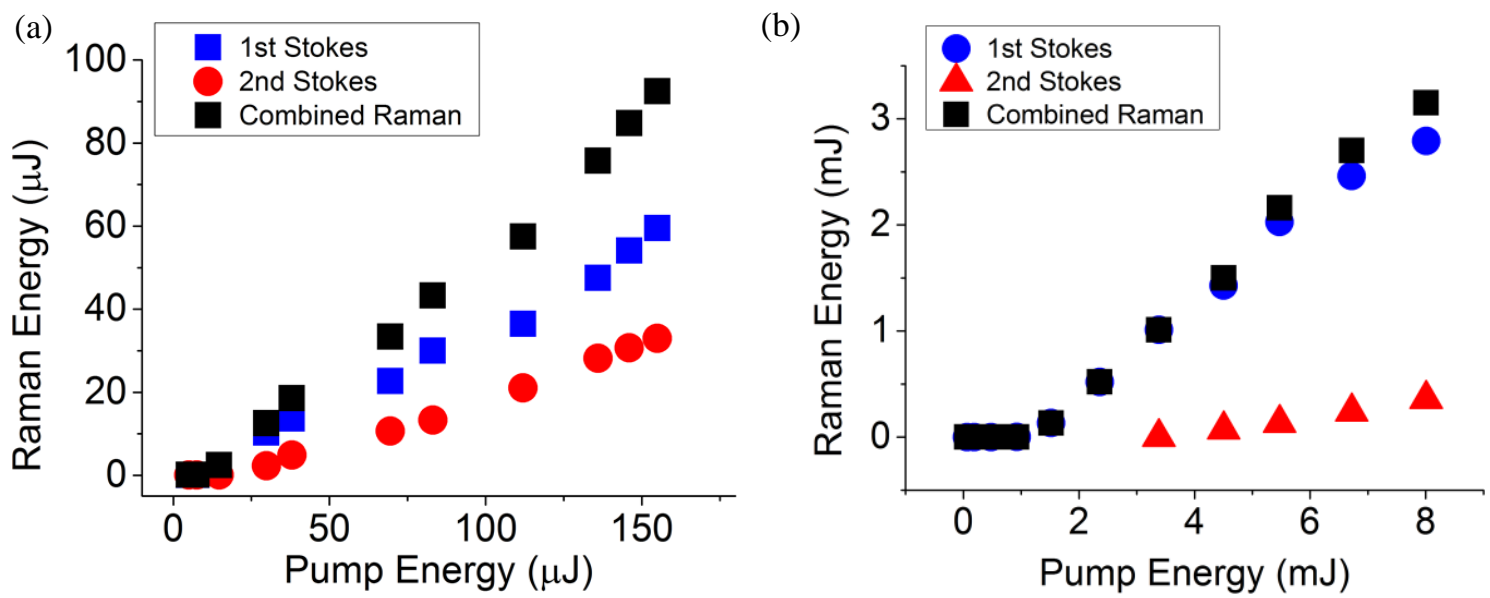

Fig. 1 Power transfer of (a) microlensed monolithic diamond Raman laser and (b) plane - plane monolithic diamond Raman laser.

\section{References}

[1] I. Friel, S. L. Geoghegan, D. J. Twitchen, and G. A. Scarsbrook, "Development of high quality single crystal diamond for novel laser applications," Proc. SPIE, vol. 7838, pp. 783819-1, 2010.

[2] S. Reilly, V. G. Savitski, H. Liu, E. Gu, M. D. Dawson, and A. J. Kemp, "Monolithic diamond Raman laser," Opt. Lett., vol. 40, no. 6, pp. 930-933, 2015 .

[3] F. Salin and J. Squier, "Gain guiding in solid-state lasers," Opt. Lett., vol. 17, no. 19, pp. 1352-1354, 1992.

[4] V. G. Savitski, S. Reilly, and A. J. Kemp, "Yellow-Orange Emission from a Plane-Plane Monolithic Diamond Raman Laser," 2015 European Conference on Lasers and Electro-Optics - European Quantum Electronics Conference, (Optical Society of America, 2015), paper CA_8_4. 